\title{
Large spectral tuning of liquid microdroplets by local heating with a focused infrared laser
}

\author{
Alper Kiraz ${ }^{a}$, Yasin Karadağ ${ }^{a}$, Saime Ç. Yorulmaz ${ }^{a}$, and Metin Muradoğlu ${ }^{b}$ \\ ${ }^{a}$ Department of Physics, Koç University, Rumelifeneri Yolu, 34450 Sariyer, Istanbul, Turkey; \\ ${ }^{b}$ Department of Mechanical Engineering, Koç University, Rumelifeneri Yolu, 34450 Sariyer, \\ Istanbul, Turkey
}

\begin{abstract}
Large deformations can easily be introduced in liquid microdroplets by applying relatively small external forces or controlling the evaporation/condensation kinetics. This makes liquid microdroplets attractive to serve as the building blocks of largely tunable optical switches or filters that are essential in optical communication systems based on wavelength division multiplexing. Solid optical microcavities have not found large use in these applications, mainly due to their rigid nature. The fact that liquid microdroplets are low-cost and disposable can also prove to be important in mass production of these photonic devices.
\end{abstract}

Here, we show that local heating with an infrared laser can be used to largely tune the whispering gallery modes (WGMs) of water/glycerol or salty water microdroplets standing on a superhydrophobic surface. In the scheme presented, a liquid microdroplet kept in a humidity chamber is stabilized on a superhydrophobic surface, and an infrared laser beam is focused near the center of the microdroplet. As a result of the local heating, the temperature of the liquid microdroplet increases, and the water content in the liquid microdroplet evaporates until a new equilibrium is reached. At the new equilibrium state, the non-volatile component (i.e. glycerol or salt) attains a higher concentration in the liquid microdroplet.

We report tunability over large spectral ranges up to $30 \mathrm{~nm}$ at around $590 \mathrm{~nm}$. For salty water microdroplets the reported spectral tuning mechanism is almost fully reversible, while for the case of glycerol/water microdroplets the spectral tuning mechanism can be made highly reversible when the chamber is saturated with glycerol vapor and the relative water humidity approaches unity.

Keywords: Microdroplet, superhydrophobic surface, whispering gallery mode, optical microcavity, spectral tuning

\section{INTRODUCTION}

Liquid microdroplets are easily deformable. They are therefore attractive as largely tunable optical microcavities for applications in optical communication systems and fundamental studies in cavity quantum electrodynamics. ${ }^{1}$ Recently we have demonstrated large spectral tuning of the whispering gallery modes (WGMs) of water microdroplets standing on a superhydrophobic surface by controlling the evaporation/condensation kinetics in a humidity chamber. ${ }^{2}$ In our demonstration the temperature profile within the humidity chamber was changed, resulting in evaporation or condensation in water microdroplets. Local control of the size of individual microdroplets was not achieved with that demonstration.

Evaporation/condensation kinetics of liquid microdroplets become much richer in case of microdroplets which are constituted of a mixture of highly nonvolatile and volatile components. In particular water/glycerol and salty water mixtures have been studied in detail as model systems. Ray and coworkers have extensively examined the evaporation and growth of water/glycerol microdroplets in humidity controlled environments. ${ }^{3-5}$ In these studies, a single water/glycerol microdroplet is levitated electrodynamically and the size change controlled by the humidity of the chamber is measured highly accurately with Mie scattering experiments. For the case of salty water microdroplets, the water activities are well documented as a function of solute weight percent. ${ }^{6}$

Further author information: (Send correspondence to A.K) A.K.: E-mail: akiraz@ku.edu.tr, Telephone: +90 2123381701

Optical Trapping and Optical Micromanipulation V, edited by Kishan Dholakia, Gabriel C. Spalding, Proc. of SPIE Vol. 7038, 70381I, (2008) · 0277-786X/08/\$18 · doi: 10.1117/12.794624 
In this article we demonstrate the spectral tuning of water/glycerol or salty water microdroplets standing on a superhydrophobic surface as a result of local heating induced by the absorption of a focused infrared laser. Under constant infrared laser illumination, individual microdroplets attain a temperature that is higher than the temperature of the humidity chamber. Being warmer than the surrounding, water in the microdroplet evaporates quickly and the microdroplet almost reaches to an equilibrium at a new state with a smaller mole fraction of water; hence its size is reduced. When the infrared laser is switched off, the temperature of the microdroplet decreases and thus its size grows by water adsorption until a thermodynamic equilibrium is reached. At the end of this process, the microdroplet almost fully recovers its original size. For the case of salty water microdroplets the presented spectral tuning mechanism is almost fully reversible. For water/glycerol microdroplets, evaporation of glycerol is the source of irreversibility in spectral tuning. For this case, the spectral tuning process can be made highly reversible when the chamber is saturated with glycerol vapor and the relative water humidity approaches unity (i.e. for relative water humidities greater than $90 \%$ ).

In addition to its potential applications in optical communication systems and cavity quantum electrodynamics, the large spectral tuning method described in this manuscript can prove to be important in characterizing liquid aerosols on a surface. For example this method can be used for direct and non-invasive characterization of the thermodynamic properties and evaporation/condensation kinetics of simple and multi-component liquid aerosols..$^{7,8}$ Our first results were presented in Ref.s. ${ }^{9,10}$ In Ref. ${ }^{9}$ the laser induced size change of the microdroplet was mistakingly interpreted to be due to the optical scattering force. This interpretation error was then corrected in Ref. ${ }^{10}$

For over thirty years, optical spectroscopy experiments have been performed on liquid microdroplets flying in air or suspended using various position stabilization techniques ${ }^{11}$ such as optical levitation, ${ }^{12,13}$ electrodynamic levitation, ${ }^{3,4,14}$ and optical tweezing. ${ }^{15}$ In the experiments reported in this article we use a superhydrophobic surface in position stabilization. In addition to preserving the sphericity of the microdroplets, the use of the superhydrophobic surface improves the robustness of the experiments against external disturbances.

\section{THEORY}

\subsection{Evaporation of a Water/Glycerol or Salty Water Microdroplet}

A simple lumped system formulation is used for mass and heat transfer between the microdroplet and the chamber. In the formulation below evaporation of water is only considered, the other component in the microdropet (i.e. glycerol or salt) is assumed to be ideally nonvolatile. In this model, we assume that the composition and temperature distributions remain sufficiently uniform within the microdroplet for all times. This is a good approximation when the heat and mass transfer within the microdroplet is much more effective than the mass and heat transfer across a microdroplet interface, i.e., Biot number is much smaller than unity. In addition, we assume that heat and mass transfer occur solely due to molecular diffusion in a quasi-stationary state, i.e., the evaporation and growth rates are sufficiently slow after a short transient period. Under these assumptions, the number of moles of water $N_{A}$ in the microdroplet of radius $a$ evolve by

$$
\frac{d N_{A}}{d t}=-4 \pi a f_{P B} D_{A} \frac{P_{A}^{0}\left(T_{\infty}\right)}{R T_{\infty}}\left(\gamma_{A} x_{A} \phi_{A}-S_{A}\right),
$$

where

$$
\phi_{A}=\left(\frac{T_{\infty}}{T_{d}}\right) \frac{P_{A}^{0}\left(T_{d}\right)}{P_{A}^{0}\left(T_{\infty}\right)}
$$

In Eqs.(1,2), $D_{A}$ is the molecular diffusivity in the gas mixture, $\gamma_{A}$ is the activity coefficient, $x_{A}$ is the mole fraction, $S_{A}$ is the relative humidity in the chamber, $R$ is the universal gas constant, $P_{A}^{0}(T)$ is the vapor pressure at temperature $T$, and $T_{d}$ and $T_{\infty}$ are the temperatures at the microdroplet interface and in the chamber far from the microdroplet, respectively. The correction factor $f_{P B}$ is introduced to account for the effects of the substrate on the mass transfer and is specified by Picknett and Bexon ${ }^{16,17}$ equation:

$$
f_{P B}(\theta)=\frac{1}{2}\left\{\begin{array}{lr}
0.6366 \theta+0.09591 \theta^{2}-0.06144 \theta^{3} & 0^{o} \leq \theta<10^{\circ} \\
0.000008957+0.6333 \theta+0.116 \theta^{2}-0.08878 \theta^{3}+0.01033 \theta^{4} & 10^{\circ} \leq \theta<180^{\circ},
\end{array}\right.
$$


where the contact angle $\theta$ is in radians. In the present study, we take $\theta=160^{\circ}$ based on the experimental results obtained for millimeter size droplets sitting on the superhydrophobic surface used here. Similarly, the temperature of the microdroplet evolves by

$$
\frac{d T_{d}}{d t}=\frac{\Delta H_{v a p, A}}{N C_{P L}} \frac{d N_{A}}{d t}-\frac{3 k_{\mathrm{eff}} V_{m}}{a^{2} C_{P L}}\left(T_{d}-T_{\infty}\right)+\frac{\tilde{Q}_{a b s} P_{i n c}}{N C_{P L}},
$$

where $V_{m}, N$ and $C_{P L}$ are the molar specific volume, the total number of moles and molar heat capacity of the microdroplet mixture, respectively, $\Delta H_{v a p, A}$ is the enthalpy of evaporation and $k_{\text {eff }}$ is the effective heat conductivity taken here as a simple area average of the heat conductivities of the air $\left(k_{a}\right)$ and the substrate $\left(k_{s}\right)$, i.e., $k_{\text {eff }}=f_{a} k_{a} A_{a}+\left(1-f_{a}\right) k_{s}$ where $f_{a}=A_{a} / A_{d}$ is the ratio of the surface area of the microdroplet that is in contact with the air and the total surface area of the microdroplet. The last term in Eq. (4) represents the heating of the microdroplet by the focused infrared laser beam. $\tilde{Q}_{a b s}$ is the ratio of the total power absorbed by the microdroplet to the total power of the incident beam $\left(P_{\text {inc }}\right)$.

For water/glucerol microdroplets, the activity coefficient of water $\left(\gamma_{A}\right)$ is computed from the van Laar equation

$$
\ln \gamma_{A}=\frac{c_{1}}{\left(1+\frac{c_{1}}{c_{2}} \frac{x_{A}}{1-x_{A}}\right)^{2}},
$$

where the parameters $c_{1}=-0.3049$ and $c_{2}=-0.8551$ are obtained from the best fit to the experimental data by $\mathrm{Tu}$ and Ray. ${ }^{3}$ For salty water microdroplets, the activity coefficient is computed using the polynomial relationship:

$$
\gamma_{A}=1.0+\sum C_{i} x_{N a C l}^{i}
$$

where $x_{\mathrm{NaCl}}$ denotes the weight percent of $\mathrm{NaCl}$, and the polynomial coefficients are $C_{1}=-6.366 \cdot 10^{-3}$, $C_{2}=8.624 \cdot 10^{-5}, C_{3}=-1.158 \cdot 10^{-5}$, and $C_{4}=1.518 \cdot 10^{-7}$ for $\mathrm{NaCl}^{6}$

\subsection{Laser Induced Heating}

Laser induced heating is computed using the localized approximation of the generalized Lorenz-Mie theory applied to the absorption of a focused laser beam by a spherical microdroplet suspended in air. ${ }^{18-20}$ The calculations are based on the correction coefficients given in Ref. ${ }^{18}$ for on-axis illumination. The normalized absorption efficiency $\left(\tilde{Q}_{\mathrm{abs}}\right)$ is plotted in Fig. 1 as a function of the structure factor $(\alpha=2 \pi a / \lambda)$ for borth water/glycerol and salty water microdroplets. In these calculations, wavelength and focal waist are taken as $\lambda=1064 \mathrm{~nm}$ and $w_{0}=1000 \mathrm{~nm}$, respectively. For salty water microdroplets the refractive index is taken as that of water, and water/glycerol microdroplets the refractive index is calculated as: $n_{L}=v_{A} n_{A}+v_{B} n_{B},{ }^{5}$ where $v_{A}$ and $v_{B}$
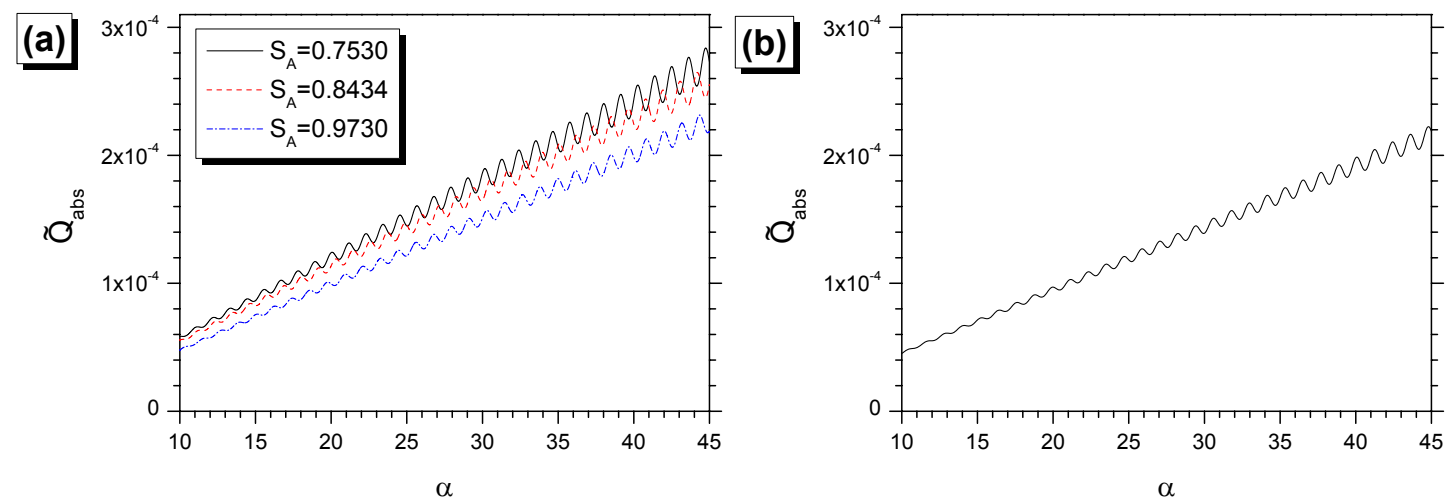

Figure 1. Normalized absorption efficiency as a function of the structure factor $(\alpha=2 \pi a / \lambda)$ for the three different relative water humidities used in the experiments. A perfectly spherical microdroplet is assumed to be suspended in air, and the effects of the superhyrophobic substrate are ignored. Low quality oscillations are due to the Fabry-Perot resonace modes. 

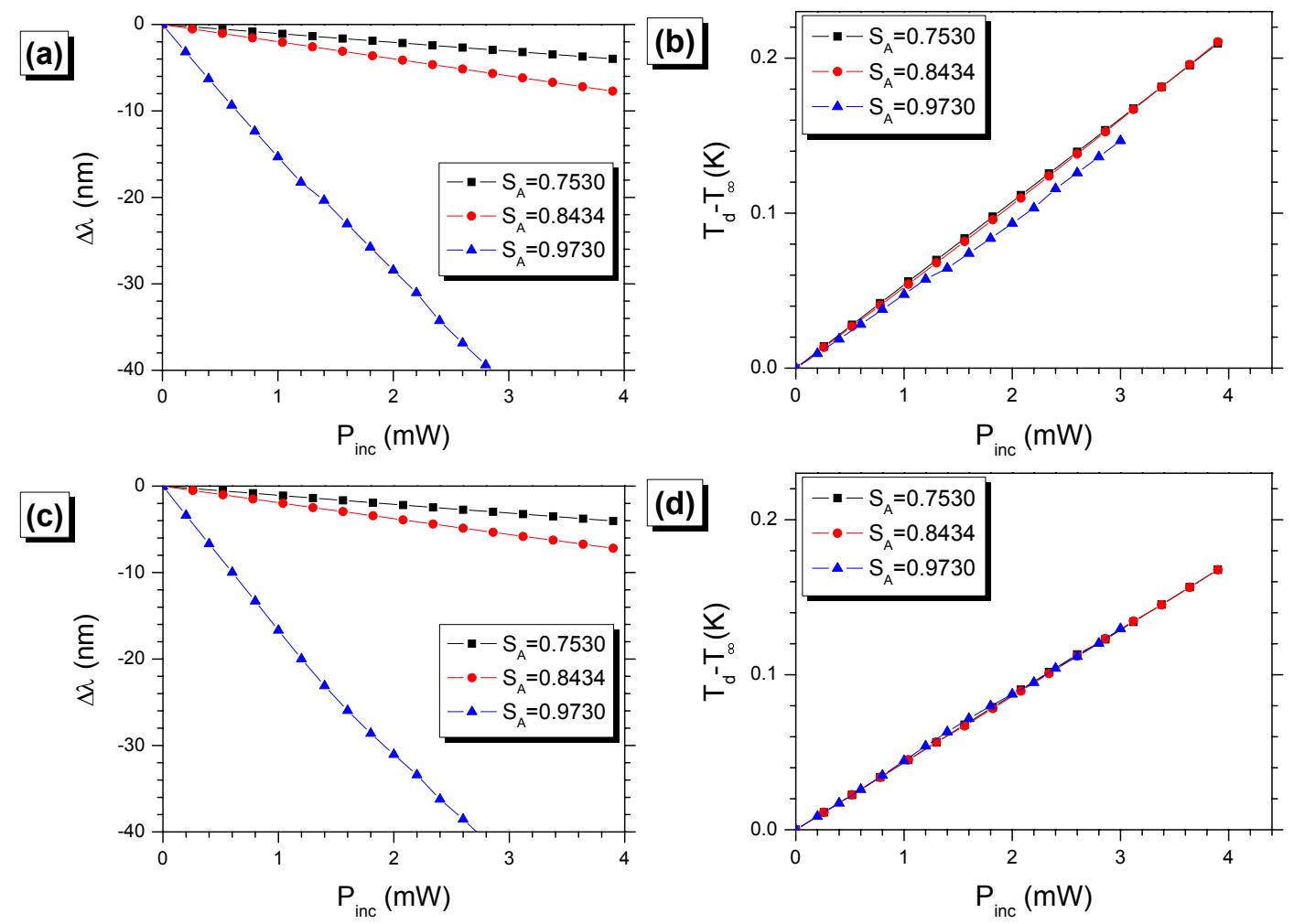

Figure 2. Results of the computations performed for water/glycerol (a-b) and salty water (c-d) microdroplets assuming $7 \mu \mathrm{m}$ microdroplet diameter. (a) and (c) show the computational spectral drifts observed in WGMs at around $590 \mathrm{~nm}$ as a function of incident laser power for three different relative water humidities. (b) and (d) show the expected temperature difference between the microdroplet and the chamber as a function of incident laser power for three different relative water humidities.

denote the volume fractions of water and glycerol, respectively. Complex refractive index of water and glycerol are taken as $n_{A}=1.33+1202.32 \cdot 10^{-9} i$ and $n_{B}=1.47+1811.95 \cdot 10^{-9} i$ assuming the absorption coefficients in Ref. ${ }^{21}$ Due to on-axis illumination, the high quality WGMs are not observed in Fig. 1 and the low quality oscillations correspond to the Fabry-Perot resonance modes. ${ }^{22}$ As expected, for large values of $\alpha$, i.e., $\alpha>50$, the present calculations almost exactly match with the absorption of a dielectric slab with a thickness of $2 a$. Note that the omission of the superhydrophobic surface is a source of error in the present calculations. A simple three dielectric layer (glass/microdroplet/air) model indicates that the omission of substrate may result in up to $5 \%$ more laser induced heating than the actual value.

\subsection{Computational Results}

Steady state solutions of the rate equations (1) and (4) are presented in Fig. 2 for both water/glycerol (a-b) and salty water (c-d) microdroplets, considering $7 \mu \mathrm{m}$ microdroplet diameter. Under a constant laser illumination, the steady state value of $T_{d}$ is larger than $T_{\infty}$ by $\tilde{Q}_{a b s} P_{i n c} a^{2} / 3 k_{g} V_{m} N$. For this value of $T_{d}, x_{A}$ is then obtained from the steady state relation $\gamma_{A} x_{A} \phi_{A}=S_{A}$. The corresponding spectral shifts in the WGMs located at around $590 \mathrm{~nm}$ are plotted as a function of the incident infrared laser power $\left(P_{i n c}\right)$ for three different $S_{A}$ values used in the experiments. The calculations in Fig.s 2a and 2c use the asymptotically linear relationship between the spectral position of the WGMs and the normalized equatorial radius, ${ }^{23,24}$ and they are valid for WGMs at around $590 \mathrm{~nm}$ irrespective of the size of the microdroplet. Our spectral drift calculations made for microdroplets with diameters ranging between $6-12 \mu \mathrm{m}$ (results not shown) show that for a given relative water humidity and incident laser power, similar spectral drifts are expected for all microdroplet sizes. Fig.s $2 \mathrm{~b}$ and $2 \mathrm{~d}$ show the expected microdroplet temperature $\left(T_{d}\right)$ as a function of the $P_{i n c}$ values used in our experiments. It is interesting 


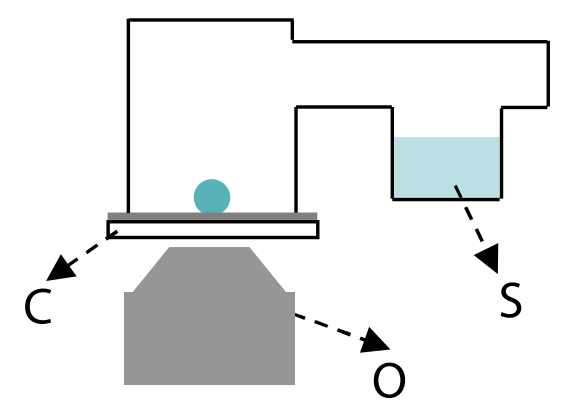

Figure 3. Illustration of the experimental setup. O: microscope objective; C: cover glass with superhydrophobic coating; S: saturated salt/water solution.

to see that the expected temperature difference between the microdroplet and the chamber remains less than $0.25 \mathrm{~K}$ for all the cases in the range of laser powers used in the experiments, and this small temperature difference causes significant spectral shifts as seen in Fig.s 2a and 2c.

\section{SURFACE PREPARATION AND EXPERIMENTAL SETUP}

Superhydrophobic surfaces were prepared by spin coating $50 \mathrm{mg} / \mathrm{ml}$ ethanol dispersions of hydrophobically coated silica nanoparticles (Evonik, Aeroxide ${ }^{\circledR}$ ) on cover glasses as reported previously. ${ }^{2,9}$ Average contact angle of millimeter-sized droplets was measured to be larger than $155^{\circ}$ on these surfaces. Water/glycerol and salty water microdroplets were generated with an ultrasonic nebulizer at room temperature and pressure. Solutions containing 5-20 $\mu \mathrm{M}$ Rhodamine B and 90/10 volume distribution of water/glycerol or $5 \mathrm{M} \mathrm{NaCl}$ are used to generate water/glycerol or salty water microdroplets. Following microdroplet generation, water content in the microdroplets quickly evaporates, resulting in microdroplets with diameters ranging from a few up to $20 \mu \mathrm{m}$. Superhydrophobic surface containing the microdroplets is then placed in the humidity chamber having a controlled relative humidity. After waiting for the growth of the microdroplets to their equilibrium sizes for several hours, optical experiments are performed.

The humidity chamber used in the experiments is shown in Fig. 3. A saturated salt/water solution is kept in the humidity chamber in order to have a high and stable relative humidity. Saturated water solution of three different salts are used in the experiments: (I) $\mathrm{NaCl}$, (II) $\mathrm{KCl}$, and (III) $\mathrm{K}_{2} \mathrm{SO}_{4}$. Calibrated relative humidities are $S_{A}=0.7530, S_{A}=0.8434$, and $S_{A}=0.9730$ for cases I, II, and III respectively. ${ }^{25}$

A home-made continuous wave $(\mathrm{cw})$ infrared solid state laser $(\lambda=1064 \mathrm{~nm}$, maximum output power: $\sim 1 \mathrm{~W})$ is used for local heating of microdroplets. The infrared laser is transmitted through a $2 \mathrm{x}$ telescope, a continuously variable neutral density filter, a neutral density filter used as a beam combiner, reflected by a dichroic mirror, and focused to the center of individual microdroplets using a high numerical aperture microscope objective (NA=1.4 oil, 60x), in the inverted geometry. WGMs are observed by recording the Rhodamine B emission spectra collected from individual microdroplets upon their excitation by a cw green solid state laser $(\lambda=532 \mathrm{~nm})$. The green laser is transmitted through a $6 \mathrm{x}$ telescope, reflected by the neutral density filter used as the beam combiner and the dichroic mirror, and focused approximately to the rim of the microdroplets. Rhodamine B emission is collected with the same microscope objective, transmitted through the dichroic mirror and a 1.5x magnification element, dispersed by a $50 \mathrm{~cm}$ monochromator (600 grooves/mm grating), and detected by a CCD camera using an exposure time of $0.5 \mathrm{sec}$ with a spectral resolution of $0.17 \mathrm{~nm}$. A mechanical shutter is used to block the green laser during the unexposed periods, while the infrared laser is always illuminating the microdroplets at varying intensities controlled with the continuously variable neutral density filter. Excitation powers at the focus of the microscope objective are $\sim 1 \mu \mathrm{W}$ for the green laser and between $0-5 \mathrm{~mW}$ for the infrared laser.

\section{RESULTS AND DISCUSSION}

Fig. 4a shows examplary emission spectra obtained from a $7.0 \mu$ m diameter water/glycerol microdroplet during spectral tuning experiments performed under a relative water humidity of $S_{A}=0.8434$. The incident laser power 

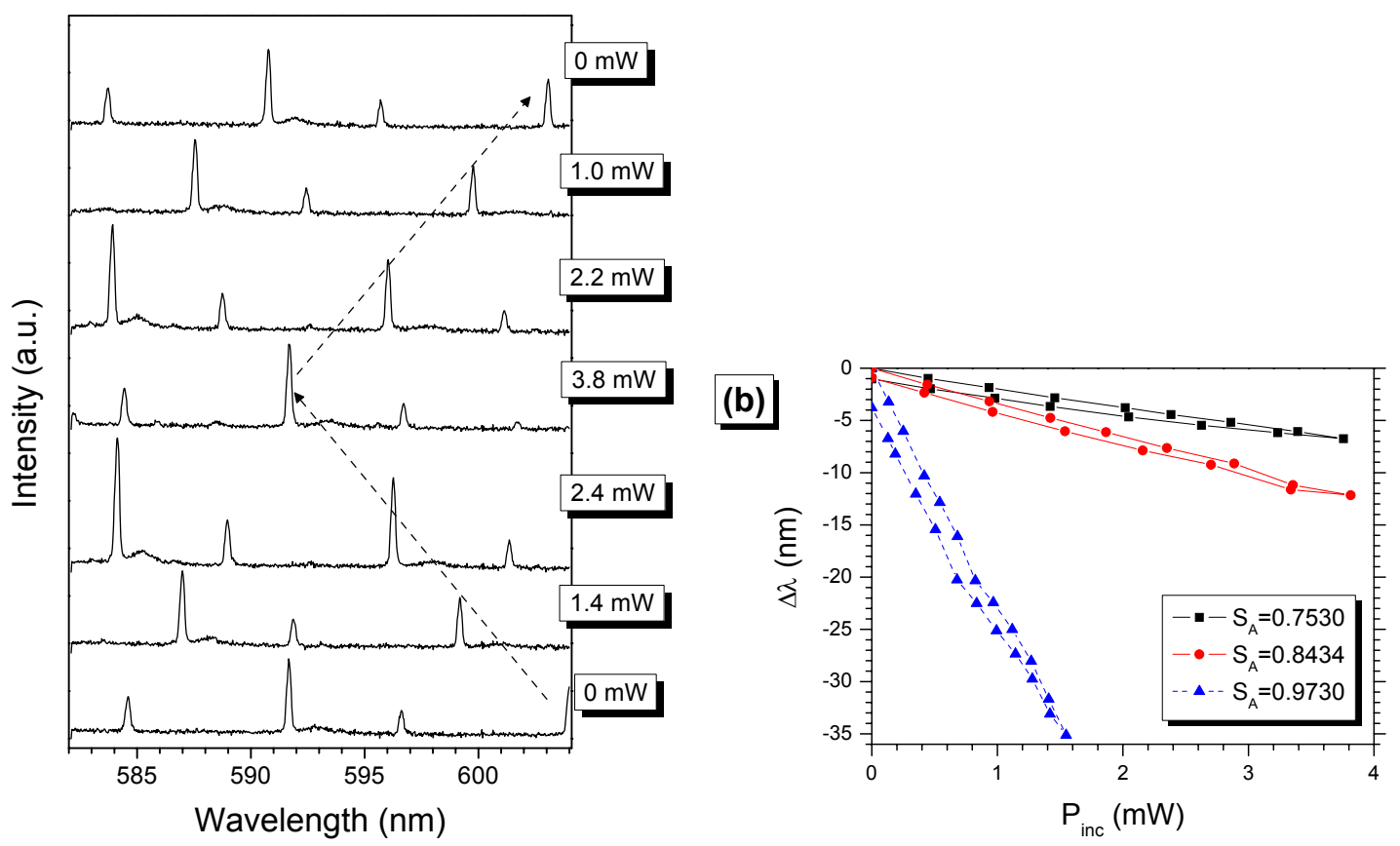

Figure 4. (a) Emission spectra recorded from a $7.6 \mu \mathrm{m}$ diameter water/glycerol microdroplet while the infrared laser power is first increased from and then decreased to zero. Arrows indicate the spectral drift direction of the high quality WGMs belonging on the same mode set. At the maximum infrared laser power of $3.8 \mathrm{~mW}$, total spectral drift is $12.2 \mathrm{~nm}$. (b) The reversibility of spectral tuning observed in three water/glycerol microdroplets for three relative water humidities. Microdroplet diameters are $6.4,7.0$ and $7.0 \mu \mathrm{m}$ for $S_{A}=0.7530, S_{A}=0.8434$ and $S_{A}=0.9730$, respectively.

is first gradually increased from zero to the maximum power of $3.8 \mathrm{~mW}$ resulting in the blue shift of the WGMs. The incident laser power is then gradually decreased to zero resulting in the red shift of the WGMs. Arrows in this figure indicate the spectral drift of a high quality WGM having a resolution limited spectral width of $0.17 \mathrm{~nm}$. A total spectral drift of $12.2 \mathrm{~nm}$ is observed in the WGMs at the maximum infrared laser power of $3.8 \mathrm{~mW}$. At the end of the experiment, WGMs are observed to be blue shifted by $0.9 \mathrm{~nm}$ compared to third spectral positions at the beginning of the experiment, because of the evaporation of glycerol. The high quality lineshape function of the WGMs are well preserved during spectral tuning. This indicates that the sphericity and the contact angle of the microdroplet did not change considerably throughout the experiment. ${ }^{26}$

In Fig. 4b we present the spectral drifts $(\Delta \lambda)$ observed in WGMs of three water/glycerol microdroplets as a function of incident laser power $\left(P_{i n c}\right)$ for three relative water humidities of $S_{A}=0.753,0.8434$ and 0.973 . For all cases a nearly linear relationship is observed between $\Delta \lambda$ and $P_{i n c}$, which is in good qualitative agreement with the computational results presented in Fig. 2. In Fig. 4b the predicted spectral drifts are slightly smaller than the measured values in all three values of $S_{A}$. In addition to simplifications made in lumped system formulation, this difference is partly attributed to the omission of the superhydrophobic substrate in computing laser induced heating. In Fig. $4 \mathrm{~b}$, spectral drifts of $-1.0,-0.9$, and $-3.8 \mathrm{~nm}$ are observed at the end of the spectral tuning cycles in which maximum drifts of $-6.8,-12.2$, and $-35.1 \mathrm{~nm}$ are observed for $S_{A}=0.7530,0.8434$ and 0.9730 , respectively. Hence for $S_{A}=0.7530,0.8434$ and 0.9730 the spectral drift at the end of the tuning cycle is 14.7 , $6.6,10.8$ percent of the maximum spectral tuning observed within the cycle.

Although not presented in this manuscript, our experiments using salty water microdroplets have revealed results that are similar to those presented in Fig. 4. A nearly linear relationship has been observed between $\Delta \lambda$ and $P_{i n c}$ for three different relative water humidities, and the observed spectral drifts have been in good qualitative agreement with the computational results presented in Fig. 2. Furthermore, almost fully reversible spectral tuning has been observed in salty water microdroplets. This is in contrast to the case of water/glycerol microdroplets where irreversibility in spectral tuning is caused by the evaporation of glycerol. 


\section{CONCLUSIONS}

We studied the spectral tuning of a water/glycerol or salty water microdroplet by local heating with a focused infrared laser beam both experimentally and computationally. The tuning mechanism is based on the size change of the microdroplet controlled by focused infrared laser through evaporation and condensation of water in the microdroplet due to laser induced heating. Computational results are found to be in good qualitative agreement with the experimental measurements. It is found that highly reversible large $(>30 \mathrm{~nm})$ spectral tuning can be achieved using this technique. From the experimental and computational results, we draw the following conclusions.

1. Observed large size changes in microdroplets are mainly caused by evaporation of their water content in response to the laser induced heating.

2. Spectral tuning is essentially independent of the microdroplet size but it is very sensitive to the relative water humidity in the chamber. The sensitivity of tuning increases rapidly with increasing relative water humidity.

3. For water/glycerol microdroplets, the evaporation of glycerol is still important for reversibility of tuning mechanism. Both computational and experimental results indicate that irreversibility is mainly caused by evaporation of glycerol and reversibility can be increased dramatically when air in the chamber is saturated with glycerol vapor.

4. For salty water microdroplets, both computational and experimental results indicate that spectral tuning method is almost fully reversible.

The large spectral tuning technique presented in this article can inspire novel, largely tunable liquid microdroplet based components for optical communication systems. The presented technique can also be used in fundamental studies in cavity quantum elctrodynamics, and in characterizing liquid aerosols on a surface.

\section{ACKNOWLEDGMENTS}

This work is partially supported by the Scientific and Technological Research Council of Turkey (Grant No. TÜBİTAK-105T500). The authors thank the Alexander von Humboldt Foundation for equipment donation. A. Kiraz acknowledges the financial support of the Turkish Academy of Sciences in the framework of the Young Scientist Award program (Grant No. A.K/TÜBA-GEBIP/2006-19).

\section{REFERENCES}

[1] Vahala, K. J., "Optical microcavities," Nature 424, 839 (2003).

[2] Kiraz, A., Kurt, A., Dündar, M. A., and Demirel, A. L., "Simple largely tunable optical microcavity," Appl. Phys. Lett. 89, 081118 (2006).

[3] Tu, H. and Ray, A. K., "Measurement of activity coefficients from unsteady state evaporation and growth of microdroplets," Chem. Eng. Comm. 192, 474-498 (2005).

[4] Ray, A. K., Johnson, R. D., and Souyri, A., "Dynamic behavior of single glycerol droplets in humid air streams," Langmuir 5, 133-140 (1989).

[5] Tu, H., Application of Light Scattering in Studies of Transport, Thermodynamics, Light Absorption, and Electric Properties of Single Droplets, PhD thesis, University of Kentucky (2001).

[6] Tang, I. N., Tridico, A. C., and Fung, K. H., "Thermodynamic and optical properties of sea salt aerosols," J. Geophys. Res. 102, 23269-23275 (1997).

[7] Reid, J. P., Meresman, H., Mitchem, L., and Symes, R., "Spectroscopic studies of the size and composition of single aerosol droplets," Int. Rev. Phys. Chem. 26, 139-192 (2007).

[8] Hopkins, R. J., Howle, C. R., and Reid, J. P., "Measuring temperature gradients in evaporating multicomponent alcohol/water droplets," Phys. Chem. Chem. Phys. 8, 2879-2888 (2006). 
[9] Kiraz, A., Ç. Yavuz, S., Karadă̆, Y., Kurt, A., Sennaroglu, A., and Çankaya, H., "Large spectral tuning of liquid microdroplets standing on a superhydrophobic surface using optical scattering force," Appl. Phys. Lett. 91, 231102 (2007).

[10] Kiraz, A., C. Yavuz, S., Karadă̆, Y., Kurt, A., Sennaroglu, A., and Çankaya, H., "Erratum: large spectral tuning of liquid microdroplets by optical scattering force [appl. phys. lett. 91, 231102 (2007)]," Appl. Phys. Lett. 92, 129902 (2008).

[11] Davis, E. J., "A history of single aerosol particle levitation," Aerosol Sci. Technol. 26, 212-254 (1997).

[12] Ashkin, A. and Dziedzic, J. M., "Optical levitation of liquid drops by radiation pressure," Science 187, 1073 (1975).

[13] Ashkin, A. and Dziedzic, J. M., "Observation of resonances in the radiation pressure on dielectric spheres," Phys. Rev. Lett. 38, 1351 (1977).

[14] Arnold, S. and Folan, L. M., "Fluorescence spectrometer for a single electrodynamically levitated microparticle," Rev. Sci. Inst. 57, 2250 (1986).

[15] Hopkins, R. J., Mitchem, L., Ward, A. D., and Reid, J. P., "Control and characterisation of a single aerosol droplet in a single-beam gradient-force optical trap," Phys. Chem. Chem. Phys. 6, 4924 (2004).

[16] Picknett, R. G. and Bexon, R. J., "The evaporation of sessile or pendant drops in still air," J. Colloid Interface Sci. 79, 667-677 (1977).

[17] McHale, G., Aqil, S., Shirtcliffe, N. J., Newton, M. I., and Erbil, H. Y., "Analysis of droplet evaporation on a superhydrophobic surface," Langmuir 21, 11053-11060 (2005).

[18] Grehan, G., Maheu, B., and Gouesbet, G., "Scattering of laser beams by mie scatter centers: numerical results using a localized approximation," Appl. Opt. 25, 3539-3548 (1986).

[19] Lock, J. A., "Improved gaussian beam-scattering algorithm," Appl. Opt. 34, 559-570 (1995).

[20] Lock, J. A., "Excitation efficiency of a morphology-dependent resonance by a focused gaussian beam," $J$. Opt. Soc. Am. A 15, 2986-2994 (1998).

[21] Peterman, E. J. G., Gittes, F., and Schmidt, C. F., "Laser-induced heating in optical traps," Biophys. J. 84, 1308-1316 (2003).

[22] Cheng, D. K., [Field and Wave Electromagnetics], Addison-Wesley (1991).

[23] Qian, S.-X., Snow, J. B., Tzeng, H. M., and Chang, R. K., "Lasing droplets: Highlighting the liquid-air interface by laser emission," Science 231, 486 (1986).

[24] Datsyuk, V. V., "Optics of microdroplets," J. Mol. Liq. 84, 1308-1316 (2001).

[25] Lide, D. R., $[C R C$ Handbook of Chemistry and Physics, Internet Version 2005, $<h t t p: / / w w w . h b c n e t b a s e . c o m>$ ], CRC Press (2005).

[26] Kiraz, A., Karadăg, Y., and Coskun, A. F., "Spectral tuning of liquid microdroplets standing on a superhydrophobic surface using electrowetting," Appl. Phys. Lett. 92, 191104 (2008). 